\title{
Vaccines, therapeutics, and diagnostics for covid-19: redesigning systems to improve pandemic response
}

\author{
Rohit Ramchandani and colleagues propose a framework to ensure essential public health tools \\ are fairly distributed in future pandemics
}

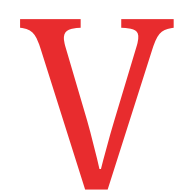

accines, therapeutics, and diagnostics are key public health tools for controlling the covid-19 pandemic, yet many countries, particularly low and middle income countries (LMICs), have had inadequate access. Many have yet to determine, for example, when and where they will be getting their vaccines from, and only $3 \%$ of people in low income countries had received at least one dose by October $2021 .^{1}$

The Access to COVID-19 Tools Accelerator (ACT-A) is a multilateral coordination mechanism set up to accelerate development, production, and equitable access to covid-19 tests, treatments, and vaccines globally. The concept brings together governments, scientists, businesses, civil society, philanthropists and global health organisations. Despite its bold vision, ACT-A has thus far fallen short of its

\section{KEY MESSAGES}

- Global efforts have been unsuccessful in providing equitable access to covid-19 vaccines, therapeutics, and diagnostics

- A core underlying issue has been a lack of shared vision that these essential public health tools should be considered a "global health commons"

- Future systems for vaccines, therapeutics, and diagnostics should be prenegotiated among countries, manufacturers, and international institutions

- Future systems should be reshaped to ensure equitable access is considered from design through to manufacturing and procurement processes

- Agreements around technology transfer and intellectual property licensing, regional trial networks, inclusive governance, and substantial predictable financing are essential expectations. Covax, the vaccines pillar of ACT-A, has been responsible for less than $6 \%$ of the 6.82 billion covid- 19 vaccines administered as of October 2021. ${ }^{23}$ The first outstanding efficacious treatment to which ACTA's therapeutic pillar has contributed is molnupiravir, which was shown to reduce the risk of hospital admission or death in October 2021. ${ }^{4}$ Oxygen therapy, still one of the best treatments available for severe cases, initially struggled from a lack of strategic prioritisation despite its critical importance. Testing did receive political attention but has been a bottleneck in many countries. $^{5}$

\section{Value chain analysis}

We used a value chain framework to analyse the successes, challenges and lessons for key covid-19 tools (table 1). ${ }^{9}$ A value chain is a business model that describes the full range of interdependent activities needed to bring a product from conception to end user. ${ }^{10}$ It can be thought of as an ecosystem of players, processes, information, and resources required to effectively deliver a product. ${ }^{1112}$ Aspects of the value chain considered included overall governance and coordination, research and development, manufacturing at scale, procurement, allocation and delivery.

\section{Governance and coordination}

While ACT-A shows unprecedented collaboration among key institutions, it has been perceived by some countries and civil society organisations as being too supply driven without enough focus on inclusivity and transparency. A lack of engagement with China, Russia, and many other LMICs, as well as delayed participation from the US government, limited the scope and acceptance of ACT-A as a global mechanism. The mechanism established itself during the emergency, in a stepwise fashion. The process was based on a standard market model-that is, development, trials, emergency use authorisation, selling to high income countries, then redistributing to
LMICs-rather than with an "end-to-end" view whereby equitable access is considered from the beginning-during product design and allocation, research and development, manufacturing, and procurement processes. It relied on the decision making mechanisms of existing organisations, which some perceive as being overly donordriven. $^{13}$

\section{Shared vision and industry policy}

Stakeholders have not had a shared vision that the measures needed to control pandemics should be considered as part of a "global health commons," available for all countries to ensure collective protection. ${ }^{6}$ A global health commons builds on the concept of a commons as managed pooled public access to certain property rights as well as access to goods and services that are valuable but in constrained supply.

The technologies to produce vaccines, diagnostics, and therapeutics are critical public health interventions to respond to international public health crises such as pandemics. Global common access to such technologies recognises this importance and the need for intervention to ensure equitable public access. Global health commons for vaccines have historically given rise to concerns about "free riders"individuals benefiting from the commons through herd immunity of others' vaccination while avoiding any potential costs of vaccination. However, a global health commons approach-recentring public health solidarity over corporate interests-has the potential to alleviate factors contributing to vaccine hesitancy or resistance.

Without this shared vision, and given the urgency in which decisions had to be made, the usual market based approach was taken. Global corporations developed and sold proprietary products designed for wealthy countries, leaving the rest of the world reliant on the goodwill of donors, development assistance, and charity. ${ }^{7}$ This is despite the substantial public investment 


\begin{tabular}{|c|c|c|}
\hline & Successes & Challenges \\
\hline $\begin{array}{l}\text { Overall governance } \\
\text { and coordination }\end{array}$ & $\begin{array}{l}\text { - ACT-A demonstrates an unprecedented collaboration by } \\
\text { multilateral agencies and the private sector } \\
\text { - WHO focus on coordination and norms }\end{array}$ & $\begin{array}{l}\text { - Mainly supply driven } \\
\text { - Western biased, led by a few donors; few LMIC and civil society voices } \\
\text { - Lack of intergovernmental decision making and shared vision of tools as "global } \\
\text { - health commons" } \\
\text { - Limited info flow from or to countries } \\
\text { - Unclear roles-eg, role of World Bank } \\
\text { - Absence of US in leading } \\
\text { - No/late China, Russia engagement }\end{array}$ \\
\hline Procurement & $\begin{array}{l}\text { - } 4.69 \text { billion doses secured (or optioned or received as } \\
\text { donation) by Covax } \\
\text { - Regional procurement happening in LMICs (eg, by African } \\
\text { Union, PAHO for Latin America and Caribbean, etc }{ }^{7} \text { ) } \\
\text { - World Bank stepped up to fund vaccines and delivery (up to } \\
\$ 20 \text { bn) }\end{array}$ & $\begin{array}{l}\text { - } 51 \% \text { of vaccine doses were reserved by countries as early as September } 2020 \\
\text { - Canada, UK, Australia, New Zealand, EU, and US secured }>200 \% \text { population } \\
\text { coverage worth of vaccine doses, leaving insufficient for LMICs and Covax } \\
\text { - Covax expects around } 1.9 \text { billion doses to be available to } 92 \text { LMICs before } \\
\text { the end of } 2021 \text {, covering roughly } 27 \% \text { of their population, well short of the } \\
\text { coverage required to control the pandemic } 8\end{array}$ \\
\hline $\begin{array}{l}\text { Allocation and } \\
\text { delivery }\end{array}$ & $\begin{array}{l}\text { - Established allocation and dose sharing principles } \\
\text { - } 2 \text { new bodies (Independent Allocation of Vaccines Group (IAVG) } \\
\text { and Joint Allocation Taskforce (JAT)) involved in allocation of } \\
\text { Covax Facility vaccines }\end{array}$ & $\begin{array}{l}\text { - Limited transparency for countries about allocation and timing of doses, leading } \\
\text { to slow delivery preparation by countries } \\
\text { - Lack of expertise in, and transparency to support, price negotiation in LMICS } \\
\text { - New challenges-supply chain, dual shot, emerging variants because of } \\
\text { inadequate vaccination coverage, infodemic, and hesitancy } \\
\text { - Inadequate and unequal investment in public health infrastructure and rollout } \\
\text { readiness } \\
\text { - Slow disbursement of committed World Bank funds } \\
\text { - Only } 3 \% \text { of people in LMICs had received at least one dose by } 21 \text { October } 2021\end{array}$ \\
\hline
\end{tabular}

before and during the pandemic in research, advanced purchases, liability waivers, and the logistics and delivery of vaccinations. In this respect, a shift to a global health commons for pandemic countermeasures seeks to realign public investment with direct common public benefit.

\section{Financing}

Insufficient and delayed financing is at the core of the failure to secure global access to life saving health technologies. It affects all parts of the value chain for each product type. Covax was unable to compete with high income nations with greater purchasing power or hosting big manufacturers. ${ }^{8}$ The funding shortage has been even more serious for therapeutics and diagnostics. For example, the ACT-A diagnostics pillar was unable to use a volume guarantee it secured for quality antigen rapid diagnostic tests because of lack of funding.

\section{Research and development}

No end-to-end strategy

Although the development of vaccines has been an extraordinary success, the global effort by Covax to make them available across populations had no clear global strategy. For example, research efforts were focused on making vaccines available as fast as possible rather than on adherence to target product profiles that consider use in LMICs (such as not requiring ultra-cold chain handling).

The lack of a clear strategy to guide research and manufacturing also applies to diagnostic tests. The development and use of target product profiles could have established the type of tests wanted, including specifications for sensitivity and specificity, early on.

\section{Weak trials with excessive focus on speed}

Weak capacity and coordination of clinical trials ${ }^{14}$-including a lack of well distributed regional trial sites, the absence of trials directly comparing effectiveness of currently available vaccines, ${ }^{15}$ and poor integration of therapeutic research pipelines into trials-has resulted in many poor quality trials with limited actionable findings.

Vaccine developers selected specific and differing endpoints for their initial trials, which in some cases facilitated faster registration. They were conducted in selected populations with limited numbers of participants in high risk groups such as elderly people and those with serious underlying medical conditions. Until at least October 2020, none of the trials was designed to detect a reduction in clinically and public health salient outcomes such as viral transmission, hospital admissions, use of intensive care, or deaths. Nor were the vaccines being studied to determine whether they could interrupt transmission of the virus. ${ }^{16}{ }^{17}$ Also, as the vaccines were researched in an extremely short space of time, safety could not be assessed reliably. While regulators around the world changed processes to speed research and regulatory pathways, many opportunities were missed for integration of improved public health criteria in selection of endpoints ${ }^{18}$ and, more generally, for greater efficiencies, better use of tools, improved data sharing, and more coordinated approaches.

Financing and institutional leadership Financing and support by the US Biomedical Advanced Research and Development 
Authority (BARDA) and the Coalition for Epidemic Preparedness Innovations (CEPI), as well as past scientific investments contributed substantially to the successful development of vaccines. However, high income countries directly funded vaccine development to help secure supply for themselves, ${ }^{19}$ which weakened CEPI's and Covax's ability to negotiate with manufacturers. By contrast, therapeutics have suffered from lack of a leading institution like CEPI as well as structural underinvestment, and the diagnostics pillar has also been significantly underfunded.

\section{WHO's role}

WHO's research and development blueprint served to establish a common research agenda quickly. It was most effective in supporting vaccine product development and to a lesser extent for diagnostics. Despite the success of the Solidarity trial for therapeutics, the experts we interviewed from other international institutions and nongovernmental organisations considered management of clinical research programmes should not be part of WHO's purview as this can create conflicts of interest given WHO's role in approving products based on trial results.

\section{Manufacturing}

Manufacturing shortages and geographical concentration of manufacturing capacity have been a key challenge. Limited transfer of technology and patent licensing have been central to these geographical limitations. Platforms and tools to enable technology transfer, such as the covid-19 technology access pool (C-TAP) and the WHO vaccine technology transfer hub have not been effectively used. The intellectual property right TRIPS waiver proposed by South Africa and India was not supported by several high income countries.

No agency is mandated to finance and strengthen manufacturing capacity for vaccines, therapeutics, and diagnostics. Expanding regional capacity for key platform technologies (eg. monoclonal antibodies and mRNA) to avoid reliance on few manufacturers and fortify supply systems should be a priority. It requires transfer of highly specific and specialised technology and know-how, in coordination with regulatory oversight, robust participation of vaccine developers, and application of good, consistent, laboratory biological manufacturing practices, and addressing financial sustainability of such facilities. Given the large challenges, a strong system is required to accelerate progress.

\section{Procurement}

Roles and responsibilities of global institutions in procuring covid-19 tools have been unclear, and regional institutions have emerged as an important procurement mechanism (box 1). There were also challenges in procuring raw materials ${ }^{22}$ and reagents necessary to implement vaccinations and diagnostics.

\section{Allocation and delivery}

In addition to the inequities highlighted above, countries say that they are receiving information on vaccine allocation without knowing when doses will arrive. This makes the planning of vaccination extremely difficult and slows down the preparation for vaccinations, including the use of funds from the World Bank and other institutions. This is increasing the risk of countries not being prepared when large quantities of doses arrive in late 2021 and across 2022, as planned by Covax and other sources.

The strategic phases and current allocation mechanism for Covax establishes two phases of vaccine allocation to recipients. Phase 1 consists of the proportional allocation of $20 \%$ of the population until all countries are covered to this level. This was intended to end the acute phase of the pandemic by securing enough doses to ensure health workers, elderly people, and vulnerable groups were protected by the end of 2021. Phase 2 will take a more epidemiological approach, consisting of weighted allocation dependent on the proportional coverage requested by countries and consideration of vulnerability and covid-19 threat. $^{23}$ Flexibility tailored to epidemiology in allocation of vaccines, therapeutics, and diagnostics is critical but requires sophisticated country level data collection and flows.

LMICs, particularly in Africa, are experiencing substantial difficulties with distribution, administration, and uptake (including from vaccine hesitancy). This highlights the importance of preparedness and systems building outside emergencies. High level political dialogues on vaccine supply and deployment have not taken place at global level despite their critical importance.

\section{Proposed framework for future mobilisation of pandemic tools}

Lessons from ACT-A can guide the establishment of a permanent platform that stands in readiness for future pandemic responses. We propose a framework that highlights five key elements of a pre-negotiated system that would accelerate research and increase the likelihood of equitable access under a global health commons (fig 1).

\section{End-to-end research and development} platform

The current supply driven research and development process needs to shift towards more of an end-to-end process in which initial research, clinical trials, manufacturing, and procurement are guided by a goal and strategy of equitable and effective access to a global health commons. Box 2 highlights two important examples.

New industry policies and country agreements At the vaccines roundtable conducted as part of the work of the Independent Panel on Pandemic Preparedness and Response, there was consensus that the international covid-19 response failed to direct industry towards equitable access and effective use of vaccines in the face of vaccine nationalism and profiteering. The same problem could arise with treatments for covid-19 and in future pandemics. Systems need to incorporate rules and industrial policies to deliberately govern the collaboration between public and private sectors (box 3). The proposed pandemic treaty should provide a platform for such rules and policies. This must cover incentives and financing, and clarify roles, responsibilities, and liabilities.

\section{Operating through regional platforms}

The future pre-negotiated system should shift to trials, manufacturing, procurement, and country coordination and collaboration through decentralised regional platforms. High income countries and emerging economies are investing in vaccine manufacturing capacity in response to the covid-19 pandemic. Regional networks should be created by piggybacking on such investments to secure a portion of manufacturing capacity for a global response mechanism and aggregate global manufacturing capacity.

The new global mechanism must also support the strengthening of the manufacturing capacity in LMICs. For example, African Union and Africa Centres for Disease Control and Prevention established the Partnership for African Vaccine Manufacturing. They call for mRNA platforms for vaccines in Africa and aim to establish at least five regional vaccine production hubs as well as fill and finish capacities. ${ }^{25}$ Difficult challenges ahead include aggregating reliable demand to meet competitive economies 


\section{Box 1: Need for clear coordinated playbooks}

- Regional institutions have emerged as important for pooled procurement and negotiation, information sharing, technical assistance, and rapid response to country needs. The Africa CDC and Africa Union's AVATT (Africa Vaccine Acquisition Task Team), for example, exemplified these functions. There are strong country demands for such regional capacity as many countries, particularly low and middle income countries, do not have access to technical and market expertise and reliable information to use when negotiating with manufacturers. In some instances, governments have been asked to put sovereign assets such as embassy buildings, military bases, or federal bank reserves as guarantees against the cost of future legal cases. ${ }^{2021}$ Regional capacity to effectively negotiate these proposals and the like, has been lacking in Asia and there is room for further strengthening in Africa and Latin America. Such capacity could alleviate some of the serious inequities in procurement and manufacturing challenges

- Gavi, the Vaccine Alliance, has had a critical role in Covax for vaccine procurement. However, there are no well funded institutions with procurement mandates for therapeutics and diagnostics. The Global Fund has been helping to fill this gap, deploying $\$ 1$ bn through its covid-19 response mechanism. It also secured substantial new funding ( $\$ 3.7 \mathrm{bn})$ to help meet procurement needs. But clarity in institutional leadership and strengthening of expertise are required for future pandemics.

- The World Bank funded country procurement of scarce commodities with a funding commitment of $\$ 6 \mathrm{bn}$ for initial response and $\$ 20 \mathrm{bn}$ for vaccine response. At the same time ACT-A pillars tried to centralise procurement with equitable distribution. This created competition between the two, and the lack of coordination hindered response efforts. Further clarity on the roles of the World Bank and other development banks, including potential reforms of their funding approach are needed. This will require an objective assessment of the bank's support for covid-19 responses including a review of options for it to finance global public goods.

of scale, access to finance, regulatory strengthening and harmonisation across countries, talent and know-how, and infrastructure. Regional institutions, WHO, and the private sector should jointly develop plans for each region, with commitments on technology transfer, supported financially by regional and other development banks.

In addition, as discussed above, regions emerged as an important unit for pooled procurement, information sharing, and rapid response to country needs. The African Union is advanced in building some

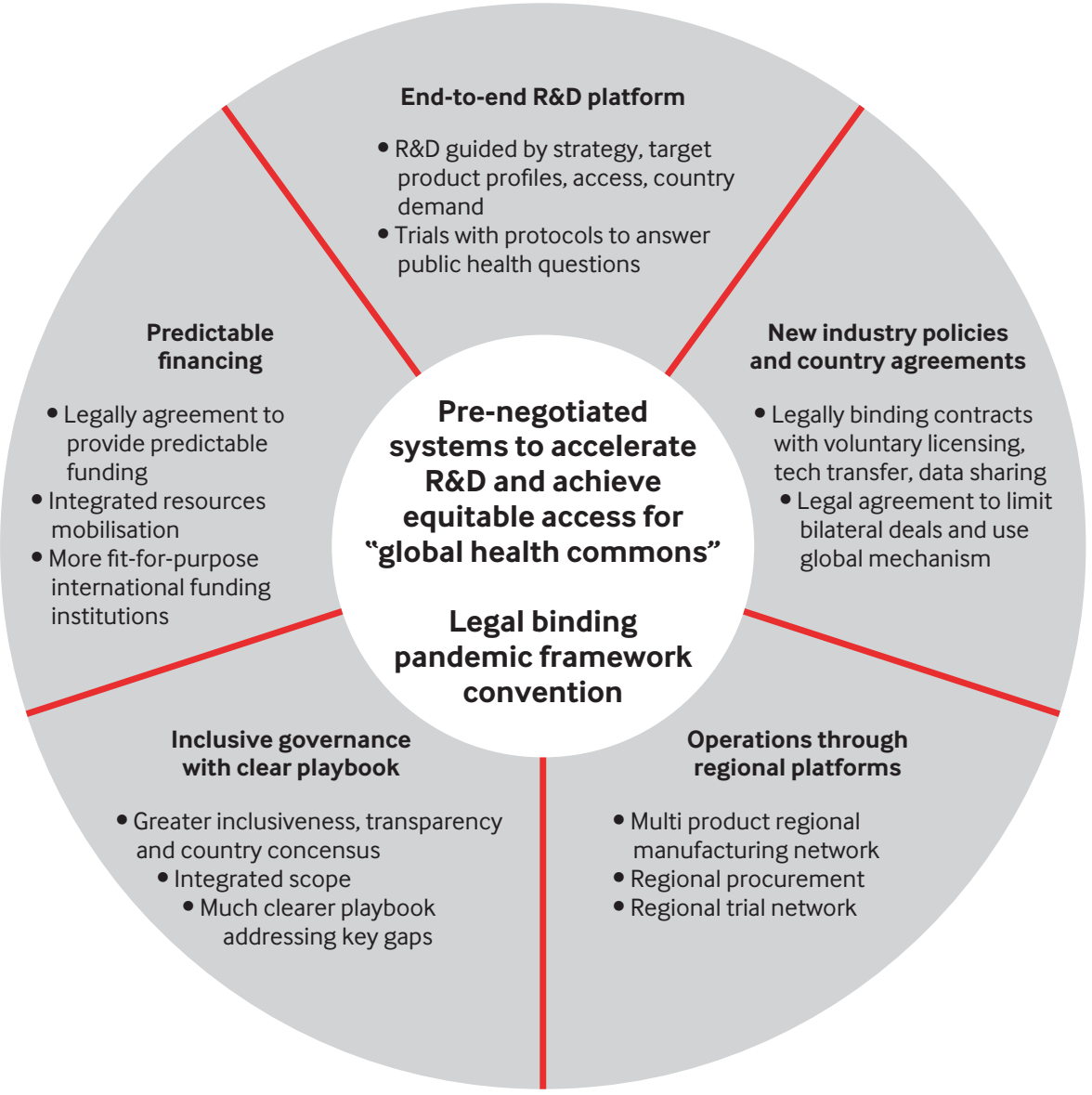

Fig 1 | Proposed five element framework for future pre-negotiated systems for a "global health commons" approach to mobilise pandemic tools

of these systems and capacities, and similar mechanisms and capacity are needed in other regions.

Clinical trial sites need to be well distributed, including in LMICs. The future global mechanism should work with regional institutions to map out, strengthen, and track the potential research network. This could also provide critical research capacity for other public health purposes (eg, poverty related and neglected tropical diseases) outside pandemics.

Inclusive governance with a clear playbook The current system needs to be reshaped to include representation from low and middle income countries, civil society organisations, countries with relevant manufacturers (such as China, Russia, and India), high income countries, and private manufacturers. It also requires effective ways of communicating with stakeholders to ensure all voices are heard. The new mechanism should also establish a much clearer playbook with deliberate strengthening of key institutions to address critical gaps across the whole value chain (eg, research into therapeutics, at-risk manufacturing across all products, procurement for therapeutics and diagnostics, oxygen, protective equipment) as well as better integration of the World Bank and regional institutions and more focused roles for WHO.

\section{Predictable financing}

Equipping a new global mechanism with substantial upfront funds to counter nationalism and enable rapid response, as well as strengthening global manufacturing capacity and meeting other financing needs for pandemic preparedness, requires fund- 


\section{Box 2: Examples of end-to-end research process}

\section{Strategy-led portfolio approach}

The independent prioritisation and simultaneous development of multiple product candidates is carried out in alignment with country demand, clear testing, treatment, and vaccination strategies, as well as target product profiles. ${ }^{24}$ This minimises risks associated with product failures and facilitates a greater number of appropriate products for all settings, in particular for low and middle income countries (eg, those with easier routes of administration and storage). WHO's research blueprint for covid-19 and the coordinated global research roadmap should be developed and used further.

\section{Clinical trials guided by public health questions}

Clinical trials should answer the right public health questions based on high quality, standardised protocols rather than being focused on obtaining regulatory approval. Trials should directly compare products. This requires effective central coordination across trials; prioritising what candidates to test; managing partnerships with regulators and manufacturers; and making data transparent through a data sharing hub. ing on a much greater scale than currently occurs. The Independent Panel recommended creating a new financing facility for pandemic preparedness and response. The facility should be built on long term (10-15 year) annual contributions from countries based on ability to pay, with larger and wealthier economies paying the most, preferably in addition to current overseas development commitments.

Making the funds already available from international financing institutions more fit for purpose during pandemics would also be critical. For example, tensions have occurred between the World Bank's country financing and global pooled funding through ACTA. The bank should assess its financing approach to covid-19, including vaccines and essential supplies, and reconfigure it to make the most out of such large funding. This should include considering its use of loans and how these affect countries' rapid use of funds, as well as countries' debt situation. The results can inform the efforts of other development banks.

These proposed shifts are inextricably linked. Missing any one element risks undermining an effective solution. Many of the reforms-such as inclusive governance and clear legal agreements on financing and industry policies-should be initiated now, leveraging the opportunities of additional ACT-A financing and a pandemic treaty, whereas others, such as regional manufacturing networks, will be longer term. Implementing these reforms will require commitment from the highest levels of governments, industry, and multilateral institutions. This can be formulated as part of a legally binding pandemic treaty or framework convention. Country leaders must act now to prevent serious inequalities in access to pandemic tools from happening again.

We thank the Independent Panel for Pandemic Preparedness and Response secretariat and all the experts interviewed as part of the data collection process.

Contributors and sources:This paper is based on analysis that was part of the work commissioned by the Independent Panel for Pandemic Preparedness and Response. Data were collected through literature review of peer reviewed papers, policy documents, public reports, and articles; semistructured interviews with 25 experts representing multilateral, philanthropy, research, non-governmental organisations, and country (high, low, and middle income) perspectives related to covid-19 tools; and

\section{Box 3: Key elements of the industry policies and country agreements}

- Pre-negotiated contracts-As part of the new global mechanism, pre-negotiate legally binding contracts for pandemic emergencies with key manufacturers, based on an agreed trigger. The contracts could include one-off or real time manufacturing capacity for diagnostics, therapeutics, or vaccines for the global mechanism and provision of voluntary intellectual property licences and technology transfer to generic manufacturers. This will be subject to predefined terms including adequate remuneration.

- Reshape Covax as a truly global approach to influence industry with country agreementsGreater funding and more participation in Covax from high income countries before or at the start of future pandemics would enable it to secure more vaccine doses and better terms for the world (eg, volume guarantees, manufacturing capacity, favourable pricing). Agreements under the pre-negotiated system would need to include countries' commitments or obligations to obtain diagnostics, therapeutics, and vaccines from the global system and limit bilateral deals with manufacturers through a framework convention for pandemics. two roundtable discussions-one on vaccines, and the other on therapeutics and diagnostics-with 18 experts. RR and SM conceived and designed the manuscript. RR and SM drafted the manuscript with inputs from all authors. All authors contributed to revising the manuscript and approved the final version.

Competing interests: We have read and understood BMJ policy on declaration of interests and have no relevant interests to declare. The secretariat of the Independent Panel for Pandemic Preparedness and Response is independent.

Provenance and peer review: Commissioned; externally peer reviewed.

This collection of articles was proposed by The Independent Panel for Pandemic Preparedness and Response. Open access fees were funded by WHO and Singapore's National Medical Research Council (NMRC/ CG/C026/2017_NUHS). The BM/ commissioned, peer reviewed, edited, and made the decision to publish these articles. Kamran Abbasi was the lead editor for The BMJ.

Rohit Ramchandani, professor 1,2,3,4

Michel Kazatchkine, panel member

Joanne Liu, panel member ${ }^{3,6}$

Preeti Sudan, panel member ${ }^{3}$

Mark Dybul, panel member ${ }^{3,}$

Precious Matsoso, panel member

Anders Nordström, head of secretariat ${ }^{8}$

Alexandra Phelan, panel secretariat member ${ }^{7,8}$ Helena Legido-Quigley, associate professor ${ }^{8,9}$ Sudhvir Singh, panel secretariat member 8,10

Shunsuke Mabuchi, panel secretariat member ${ }^{2,8}$

${ }^{1}$ Antara Global Health Advisors, Toronto, Canada ${ }^{2}$ Johns Hopkins University Bloomberg School of Public Health, Baltimore, USA

${ }^{3}$ University of Waterloo School of Public Health Sciences, Waterloo, Canada

${ }^{4}$ Balsillie School of International Affairs, Waterloo, Canada

Independent Panel for Pandemic Preparedness and Response

${ }^{6}$ McGill University's School of Population and Global Health, Montreal, Canada

7 Georgetown University, USA

${ }^{8}$ Independent Panel for Pandemic Preparedness and Response Secretariat

${ }^{9}$ Saw Swee Hock School of Public Health, National University of Singapore, Singapore

${ }^{10}$ Faculty of Medical and Health Sciences, University of Auckland, Auckland, New Zealand

${ }^{11}$ Department of Global Health and Development, London School of Hygiene and Tropical Medicine, London, UK

Correspondence to: SMabuchi shunsuke.mabuchi@gmail.com

\section{(2) $(\mathbb{1} \Theta$ OPEN ACCESS}

This is an Open Access article distributed under the terms of the Creative Commons Attribution IGO License (https://creativecommons.org/licenses/ by-nc/3.0/igo/), which permits use, distribution, and reproduction for non-commercial purposes in any medium, provided the original work is properly cited.

\section{Check for updates}

1 Our World in Data. Coverage of at least one vaccination in LMICs. 22 Oct 2021. https:// ourworldindata.org/covid-vaccinations 
2 COVAX. Live vaccine roll out. Oct 2021. https://www. gavi.org/covax-vaccine-roll-out

3 Ritchie H, Mathieu E, Rodés-Guirao L, et al. Coronavirus (COVID-19) vaccinations. Oct 2021. https://ourworldindata.org/coronavirus

4 Mahase E. Covid-19: Molnupiravir reduces risk of hospital admission or death by $50 \%$ in patients at risk, MSD reports. BMJ 2021;375:n2422. doi:10.1136/bmi.n2422.

5 Boehme C, Hannay E, Pai M. Promoting diagnostics as a global good. Nat Med 2021;27:367-8. doi:10.1038/s41591-020-01215-3

6 Smith-Nonini S. Conceiving the health commons: operationalizing a 'right' to health. Social analysis. International Journal of Social and Cultural Practice 2006;50:233-45. https://www.jstor.org/ stable/23182122.

7 Torreele E, Kazatchkine M, Mazzucato M. Preparing for the next pandemic requires public health focused industrial policy. BMJ Opinion, 1 Apr 2021. https:// blogs.bmj.com/bmj/2021/04/01/preparing-forthe-next-pandemic-requires-public-health-focusedindustrial-policy/

8 Why a pioneering plan to distribute COVID vaccines equitably must succeed. Nature 2021:589:170. doi:10.1038/d41586-021-00044-9

9 Independent Panel for Pandemic Preparedness and Response. Background paper 5 to the main report: access to vaccines, therapeutics, and diagnostics. 2021. https://theindependentpanel.org/wp-content/ uploads/2021/05/Background-paper-5-Access-tovaccines-Therapeutics-and-Diagnostics.pdf

10 Porter ME.Competitive advantage: creating and sustaining superior performance. Simon and Schuster, 2008
11 Ramchandani R. Emulating commercial, privatesector value-chains to improve access to ORS and zinc in rural Zambia: evaluation of the Colalife trial. Johns Hopkins University, 2016, https:// jscholarship.library.jhu.edu/handle/1774.2/39229.

12 Feller A, Shunk D, Callarman T. Value-chains versus supply chains. Business Process Trends 2006:1-7. https://www.bptrends.com/publicationfiles/03-06ART-ValueChains-SupplyChains-Feller.pdf

13 Key lessons of COVID-19 for Future Pandemics. Informal roundtable discussion on vaccines with invited experts. In: Kazatchkine M (Chair), Symposium hosted virtually by the Independent Panel for Pandemic Preparedness and Response 27 Jan 2021.

14 Lurie N, Keusch GT, Dzau VJ. Urgent lessons from COVID 19: why the world needs a standing, coordinated system and sustainable financing for global research and development. Lancet 2021;397:1229-36. doi:10.1016/S01406736(21)00503-1

15 Cohen J. Saying human trials aren't enough, researchers call for comparison of COVID-19 vaccines in monkeys. Science 2020. https://www.sciencemag. org/news/2020/10/saying-human-trials-arentenough-researchers-call-comparison-covid-19vaccines-monkeys

16 Doshi P. Will covid-19 vaccines save lives? Current trials aren't designed to tell us. BM/ 2020;371:m4037. doi:10.1136/bmj.m4037

17 Henry DA, Jones MA, Stehlik P, Glasziou PP. Effectiveness of COVID-19 vaccines: findings from real world studies. Med J Aust 2021;215:149-151. e1. doi:10.5694/mja2.51182

18 Mehrotra DV, Janes HE, Fleming TR, et al. Clinical Endpoints for Evaluating Efficacy in COVID-19
Vaccine Trials. Ann Intern Med 2021:174:221-8. doi:10.7326/M20-6169

19 So AD, Woo J. Reserving coronavirus disease 2019 vaccines for global access: cross sectional analysis. BMJ 2020;371:m4750. doi:10.1136/bmj.m4750

20 Kay C. Serum's Adar Poonawalla warns of delays as US prioritizes Pfizer. Bloomberg 2021 Mar 5. https://www.ndtv.com/world-news/serums-adarpoonawalla-warns-of-delays-as-us-prioritizes-pfizer2384077?amp=1\&akamai-rum=off

21 Wadvalla BA. How South Africa is dragging its vaccine rollout back from the brink. BMJ 2021;374:n1949. doi:10.1136/bmj.n1949

22 Nguyen A. What does COVAX's latest supply forecast tell us? 2021. https://www.gavi.org/vaccineswork/ what-does-covaxs-latest-supply-forecast-tell-us

23 WHO. Covax announces additional deals to access promising covid-19 vaccine candidates; plans global rollout starting Q1 2021. 2020. https://www.who.int/ news/item/18-12-2020-covax-announces-additionaldeals-to-access-promising-covid-19-vaccinecandidates-plans-global-rollout-starting-q1-2021

24 Bulc B, Ramchandani R. Accelerating global health $R \& D$ : the role of product development partnerships. 2021. http://globaldevelopment-impact.org/ wp-content/uploads/2020/07/Accelerating-globalhealth-RD_The-role-of-product-developmentpartnerships_BBulc_RRamchandani_March2021.pd

25 Nkengasong J. Building a new public health order for Africa-and a new approach to financing it. 2021. https://www.brookings.edu/essay/support-forpublic-health-preparing-for-the-next-pandemic/

Cite this as: $B M J$ 2021;375:e067488

http://dx.doi.org/10.1136/bmj-2021-067488 